\title{
Future fracture factors-predicting failure of antiresorptive therapy
}

"Even in the best-designed trial with excellent compliance a substantial proportion of patients [with osteoporosis] in the active treatment arm suffer from incident fractures," says Adolfo Díez-Pérez, in reference to antiresorptive drugs (the standard of care). Which fractures are predictable; how can the best drug be chosen for an individual; and how should inadequate response be measured and compared in clinical trials? Data are lacking; Díez-Pérez and colleagues set out to provide some.

179 women with osteoporosis (103 'adequate responders' and 76 'inadequate responders' [incident fracture despite 1-5 years of antiresorptive therapy]) were included. "A full set of specific tests for evaluating osteoporosis, including bone density, remodeling markers and bone structure assessment by image techniques, was performed," says Díez-Pérez.
Elements predictive of treatment failure were: low levels of vitamin D (required for proper antiresorptive action); fracture prior to treatment; and microarchitectural deterioration at the proximal femur-the latter factors are both related to disease severity. These findings show, says Díez-Pérez, that current treatments for osteoporosis have a "therapeutic ceiling" and are "not fully capable of compensating for bone fragility in advanced disease". Thus, besides informing ongoing efforts to develop antiresorptive therapies, these data also emphasize the importance of early treatment of osteoporosis.

Emma Leah

Original article Díez-Pérez, A. et al. Risk factors for prediction of inadequate response to antiresorptives. J. Bone Miner. Res. doi:10.1002/jbmr.1496 